Results During the study period, 39676 scans were recorded on 21089 patients. The number of scans and patients scanned differed in relation to quintiles of deprivation, with increasing numbers of scans and patients associated with increasing area-level deprivation. Significant associations were also seen between deprivation and age at scan, age at first scan, type of CT scan, and the number of scans per patient.

Conclusion Social inequalities exist in the numbers of young people undergoing CT scans with those from deprived areas more likely to do so. This is likely to reflect the rates of injuries in these individuals and implies that certain groups within the population may receive higher radiation doses than others due to medical procedures.

\section{P1-492 PREDICTORS OF CHILDHOOD PHYSICAL ACTIVITY: THE GATESHEAD MILLENNIUM STUDY}

doi:10.1136/jech.2011.142976g.80

${ }^{1} \mathrm{M}$ Pearce, ${ }^{* 1} \mathrm{~L}$ Basterfield, ${ }^{1} \mathrm{~K}$ Mann, ${ }^{1} \mathrm{~A}$ Adamson, ${ }^{1} \mathrm{~K}$ Parkinson, ${ }^{2} \mathrm{C}$ Wright, ${ }^{2} \mathrm{~J}$ Reilly. ${ }^{1}$ Newcastle University, Newcastle upon Tyne, UK; ${ }^{2}$ Glasgow University, Glasgow, UK

Introduction Conflicting evidence exists for associations between birth weight and childhood physical activity (PA) levels. It is important to know what other, potentially modifiable, factors influence PA in children given its' association with adiposity. Our aim was to identify predictors of childhood PA levels in the Gateshead Millennium Study (GMS), a population based cohort of 1029 infants born in 1999-2000 in Gateshead, Northern England.

Methods Throughout infancy and early childhood, detailed information was collected. Assessments at age 9 yrs included body composition, objective measures of habitual PA (using accelerometers during waking hours). Mean total volumes of PA (accelerometer count per minute, $\mathrm{cpm}$ ) and moderate-vigorous intensity PA (MVPA), and the percentage of time spent in sedentary behaviour (\% SB) were quantified and direct and mediating associations analysed within path models.

Results Significant differences were seen in all three outcomes between males and females $(p<0.001)$. No direct significant associations were seen with birth weight. Increased paternal age was associated with significant increases in \% SB and decreases in cpm and MVPA $(p<0.033)$. Associations with BMI at 9 yrs were in the expected directions. Increased time spent in sports clubs was significantly associated with decreased $\% \mathrm{SB}(\mathrm{p}=0.02)$ and increased MVPA $(p=0.01)$, but not cpm $(p=0.13)$.

Conclusions Although we found no evidence for an effect of birth weight on PA, path models suggest indirect effects mediated through BMI. Having an older father appeared to have a negative impact on the child's PA levels, while participation in sports clubs increases time spent in MVPA, but not cpm.

\section{P1-493 PROBABILISTIC RECORD LINKAGE: APPLICATION IN THE POPULATION-BASED CANCER REGISTRY OF SÃO PAULO (PBCR-SP), BRAZIL}

doi:10.1136/jech.2011.142976g.81

${ }^{1} S$ V Peres, ${ }^{* 1}$ M R D 0 Latorre, ${ }^{2}$ F A S Michels, ' $\mathrm{C}$ Terra. ${ }^{1}$ School of Public Health, University of São Paulo, São Paulo, Brazil; ${ }^{2}$ Population-Based Cancer Registry of São Paulo, School of Public Health, University of São Paulo, São Paulo, Brazil

Introduction Probabilistic record linkage is an alternative to different study designs because it generates, at lower operating cost, better database completeness. The aim of this study was to assess the performance of the probabilistic record linkage between PBCR-SP for cases of stomach and intestine cancer and São Paulo death database.
Methods From 1997 through 2005, 18511 cases were recorded by PBCR-SP. From the 1997 through 2007, 767752 deaths were recorded by Mortality Information System of São Paulo city. The fetal deaths were excluded. Exceeding spaces between characters were excluded. Type and size of field, and different spellings with same phoneme were standardised. Prepositions were removed. Scores for ranking true match pairs and probabilistic record linkage was performed using Reclink 3.2 software. The descriptive statistics and Kaplan-Meier test were conducted before and after linkage.

Results Of 18511 cancer cases, of both sexes, aged between 0 and 100 years and older, 7328 were true matched pairs. There was an $85.8 \%$ gain in residential address and a $11.0 \%$ gain in death date. In 4086 cases, motheŕs name was aggregated and for all paired registries, cause of death. In the survival analysis, it was verified a $14.4 \%$ underestimation in the one-year survival probability and a $31.6 \%$ underestimation for the three-year.

Conclusion Probabilistic record linkage has shown to be effective in the identification of deaths. An expressive gain in information quality of PBCR-SP database was observed.

\section{P1-494 QUALITY OF PAP SMEAR IN THE PRIMARY CARE UNITS OF SÃO PAULO CITY, BRAZIL}

doi:10.1136/jech.2011.142976g.82

${ }^{1,2} \mathrm{C}$ R P de 0 Longo, ${ }^{1,2} \mathrm{D} \mathrm{M}$ Lopes, ${ }^{1,2} \mathrm{~S}$ V Peres. ${ }^{*}{ }^{1}$ Instituto Israelita de Responsabilidade Social - Hospital Israelita Albert Einstein, Sociedade Beneficente Israelita Albert Einstein, São Paulo, Brazil; ${ }^{2}$ Secretaria Municipal de Saúde, São Paulo, Brazil

Introduction The impact of cervical cancer on morbidity and mortality depends not only on the availability and coverage of screening, but also on the quality of the sample. The aim of this study was to evaluate the quality of Pap smear of the Primary Care Units (PCU), of São Paulo city, Brazil.

Methods This was a cross-sectional study. A sample of 478 Pap smears was selected from 12 PCU-São Paulo-Brazil, between April and September 2010. Collection date, name of the responsible physician, record number, age, pregnancy presence, and previous patient's collections, date of result, presence and epithelial classification of the sample and cytological result were collected from the "registration book". The samples were classified as satisfactory with squamous columnar junction (SCJ), satisfactory without SCJ, and unsatisfactory. Samples without epithelial classification in the "registration book" were reported as "missing". Descriptive analyses were calculated.

Results The mean age was 39 years $(\mathrm{SD}=14.2)$, median 37 years (range 14-82). $38.1 \%$ of samples were satisfactory with SCJ, $22.6 \%$ satisfactory without SCJ, $0.4 \%$ unsatisfactory and $38.9 \%$ missing.

Conclusion It is concluded that there was a low percentage of unsatisfactory samples; however, the results are unreliable, because a high percentage of samples without the epithelial classification was identified. It is necessary to improve the recording of the epithelia by professionals from PCU, because a low quality examination can lead to inadequate prevention of cervical cancer.

\section{P1-495 MEASURING THE PLACEBO EFFECT IN UNBLINDED RANDOMISED COMMUNITY TRIALS}

doi:10.1136/jech.2011.142976g.83

${ }^{1} \mathrm{~N}$ Pierse, ${ }^{*}{ }^{1} \mathrm{M}$ Keall, ${ }^{2} \mathrm{R}$ Arnold, ${ }^{1} \mathrm{P}$ Howden-Chapman. ${ }^{1}$ University of Otago, Wellington, New Zealand; ${ }^{2}$ Victoria University, Wellington, New Zealand

Introduction The double blinded randomised control trial has been developed to provide gold standard estimation of causal effects. However, in many circumstances it is impossible to design studies 